\title{
Envelhecimento e Afetividade a partir do Teste de Pfister
}

\author{
Rafael Lima Dalle Mulle (D), Sonia Regina Pasian ${ }^{1}$ (D) \\ Faculdade de Filosofia, Ciências e Letras de Ribeirão Preto, Universidade de São Paulo, Ribeirão Preto-SP, Brasil
}

\section{RESUMO}

O envelhecimento populacional demanda cuidados específicos a essa etapa do desenvolvimento. Este trabalho objetivou comparar indicadores de vivências afetivas de idosos institucionalizados e não institucionalizados, a partir do Teste das Pirâmides Coloridas de Pfister (TPC), em relação a dados normativos disponíveis para a faixa etária. Foram individualmente avaliados 50 idosos $(\mathrm{G} 1=25$ institucionalizados; $\mathrm{G} 2=25$ não institucionalizados), idade média 73,6 ( $\pm 8,3$ anos), de ambos os sexos, com escolaridade fundamental e nível econômico médio-inferior, voluntários do interior de São Paulo. Responderam a questionário sociodemográfico, Mini Exame do Estado Mental (MEEM) e TPC. As escolhas e síndromes cromáticas do TPC de G1 e G2 foram comparadas (teste $t$ de Student, $p \leq 0,05$, $d$ de Cohen), não se identificando diferenças estatisticamente significativas. No entanto, em comparação às normas, G1 apresentou diferenças estatisticamente significativas nas variáveis: azul, violeta, amarelo, marrom, cinza, Síndromes Estímulo e Incolor, apontando particularidades nas vivências afetivas de idosos associados à institucionalização.

Palavras-chave: avaliação psicológica; idosos; institucionalização; técnicas projetivas; Teste das Pirâmides Coloridas.

\section{ABSTRACT - Aging and Affectiveness According to the Pfister Test}

Population aging requires specific care. This study aimed to describe and compare indicators of affective experiences of institutionalized and non-institutionalized older adults, based on the Pfister Colored Pyramids Test (CPT), in relation to normative data available for the age group. A total of 50 older adult volunteers from the state of São Paulo, mean age 73.6 years $( \pm 8.3)$, of both sexes, with elementary education and of average-lower economic levels, were individually assessed (G1 $=25$ institutionalized; G2=25 non-institutionalized). They answered a sociodemographic questionnaire, the Mini Mental State Examination (MMSE) and the CPT. The choices and chromatic syndromes of CPT of G1 and G2 were compared (Student's t-test, $p \leq .05$, Cohen's d), with no statistically significant differences being identified. However, when compared to the normative data, G1 presented statistically significant differences in the variables: blue, violet, yellow, brown, gray, Stimulus and Colorless Syndromes. It was possible to highlight particularities in the affective experiences of the older adults associated with institutionalization.

Keywords: psychological assessment; aged; institutionalization; projective techniques; Color Pyramid Test.

\section{RESUMEN - Envejecimiento y Afectividad mediante el Test de Pfister}

El envejecimiento de la población exige atención específica en esta etapa del desarrollo. Este estudio tuvo como objetivo describir y comparar indicadores de experiencias afectivas de ancianos institucionalizados y no institucionalizados, basados en Test de las Pirámides de Colores de Pfister (TPC), en relación con los datos normativos disponibles para el grupo de edad. Se evaluaron 50 ancianos (G1=25 institucionalizados; G2=25 no institucionalizados) voluntarios del interior de São Paulo, edad media de 73,6 $( \pm 8,3$ años), de ambos sexos, con enseñanza primaria y nivel de ingresos medio-bajo. Respondieron a un cuestionario sociodemográfico, Mini Mental State Examination (MMSE) y el TPC. Se compararon las elecciones de los colores y síndromes cromáticos del TPC de G1 y G2 (test $t$ de Student, $p \leq 0.05$, $d$ de Cohen), sin diferencias estadísticamente significativas. Sin embargo, en comparación con las normas, G1 mostró diferencias estadísticamente relevangtes en las variables: azul, violeta, amarillo, marrón, gris, Síndromes Estímulo e Incolor. Siendo posible señalar particularidades en las experiencias afectivas de los ancianos asociadas con la institucionalización.

Palabras clave: evaluación psicológica; ancianos; institucionalización; técnicas proyectivas; Test de las Pirámides de Colores.

O processo de envelhecimento é um fenômeno contínuo, o qual demanda diversos ajustes físicos, psíquicos e contextuais ao longo da vida (Birren \& Schaie, 2006). De acordo com a Organização Mundial da Saúde (OMS), é classificada como idosa a pessoa com mais de 65 anos de idade em países desenvolvidos e, com mais de 60 anos de idade, nos países em desenvolvimento, entre eles o Brasil
(World Health Organization [WHO], 2015). A previsão da OMS é de que o número de idosos, em 2050, passará a representar $30 \%$ da população mundial, sendo que, pela primeira vez na história, haverá mais idosos que crianças (WHO, 2015). A parcela de idosos na população nunca foi tão substancial em todo o mundo e no decorrer de toda a história. Constitui reflexo do menor crescimento

1 Endereço para correspondência: Departamento de Psicologia - FFCLRP - USP. Avenida Bandeirantes, 3900, Monte Alegre, 14040-901, Ribeirão Preto, SP. E-mail: srpasian@ffclrp.usp.br

Artigo derivado da Dissertação de mestrado de Rafael Lima Dalle Mulle com orientação de Sonia Regina Pasian defendida em 2019 no programa de pós-graduação em Psicologia da Faculdade de Filosofia, Ciências e Letras de Ribeirão Preto da Universidade de São Paulo. 
populacional aliado a menores taxas de natalidade e fecundidade, presente em países desenvolvidos e em desenvolvimento (Menezes \& Frota, 2012).

Uma alternativa para atender às necessidades de cuidado a essa faixa etária são as Instituições de Longa Permanência para Idosos (ILPIs), em número crescente, acompanhando o seu aumento populacional. Segundo a Associação Nacional de Vigilância Sanitária (Agência Nacional de Vigilância Sanitária [ANVISA], 2017), ILPIs são instituições governamentais ou não governamentais, de caráter residencial, destinada ao domicílio coletivo de pessoas com idade igual ou superior a 60 anos, com ou sem suporte familiar. Sua efetividade no cuidado ao idoso pode ser examinada a partir de diversas perspectivas técnicas e científicas, sendo que a preocupação com possíveis influências dessa condição de moradia sobre a dinâmica psíquica dos indivíduos tem ganhado destaque na literatura científica, sobretudo no Brasil (Costa \& Mercadante, 2013; Guimarães et al., 2019; Souza \& Inácio, 2017), exigindo novas investigações nessa direção.

Em estudo de caracterização do perfil do idoso residente em ILPIs no Brasil, Nicolodi e Scortegagna (2012) identificaram como variáveis predominantes nesse contexto: maioria do sexo feminino, baixo nível de escolaridade, presença de dificuldades financeiras, vivência de conflitos nos relacionamentos familiares, escassez de atividades de lazer, além de problemáticas diversas de saúde. No entanto, esse padrão parece não caracterizar apenas idosos residentes em ILPIs, mas padrão geral dos indivíduos que atingem a velhice no Brasil, que tendem a receber reduzido amparo e enfrentar adversidades de saúde e de suporte social na realidade sociocultural do país (Neri et al., 2013).

$\mathrm{Na}$ perspectiva de examinar fatores que levam idosos a residir em ILPIs, Alves-Silva, Scorsolini-Comin e Santos (2013) realizaram revisão de literatura científica referente ao período de 2001 a 2011. Identificaram que o grau de dependência (total, parcial ou independência) para realização das atividades de vida diária configurou-se como relevante variável entre os múltiplos fatores que levam à institucionalização. Para além desses achados, os autores destacaram outro ponto de convergência na literatura científica dessa área: a necessidade de mudança na orientação da assistência oferecida a esse grupo, além de aprimoramento na qualificação de cuidadores e trabalho em equipe multiprofissional, com ênfase em sua qualidade de vida e bem-estar subjetivo.

Diante desse cenário, notam-se avanços no conhecimento relacionado à Psicologia do Envelhecimento, com descobertas a respeito de possibilidades de preservação do bem-estar dos indivíduos, reforçando o interesse de pesquisa no campo (Cachioni et al., 2017). Contudo, ainda existe pouca consistência entre achados científicos nessa área, estimulando investigações com preciso delineamento, e coleta de dados com instrumentos padronizados e válidos para a realidade nacional. Dentre os múltiplos recursos técnicos utilizados nessa área, os processos de avaliação psicológica mostram-se úteis para caracterizar e compreender necessidades e demandas de intervenções e cuidados específicos dessa fase do desenvolvimento (Fontes et al., 2015; Fonseca et al., 2014; Gonçalves, 2014). No entanto, muito da produção científica sobre idosos focaliza processos neuropsicológicos, com ênfase na avaliação de funções cognitivas e executivas (Lamas et al., 2018; Martins et al., 2019).

A revisão da literatura científica sobre as diversas formas de avaliação utilizadas com idosos institucionalizados, realizada por Lima et al. (2017), oferece importante panorama sobre o tema. Foram identificados 23 diferentes instrumentos de avaliação psicológica utilizados com idosos e destes 21 focalizavam o funcionamento cognitivo. Para além das estratégias de avaliação dos processos neuropsicológicos, os métodos projetivos de avaliação psicológica aparecem como recursos válidos e confiáveis de investigação das potencialidades psíquicas. Reichert e Scortegagna (2016) realizaram revisão sobre métodos projetivos com idosos na literatura científica nacional, evidenciando sua utilidade para elucidar questões diagnósticas e para compreender tendências e demandas inconscientes dessa faixa etária.

Dentre os instrumentos de avaliação psicológica de cunho projetivo, o Teste das Pirâmides Coloridas de Pfister (TPC) destaca-se por seu caráter lúdico, de fácil aplicação, sendo bem aceito por diferentes camadas da população, inclusive idosos. Possui variáveis relacionadas ao gerenciamento emocional (Miguel et al., 2017), visando a compreensão da dinâmica afetiva e o funcionamento cognitivo (Silva \& Cardoso, 2012).

O TPC foi utilizado em investigações realizadas com idosos no Brasil (Bastos-Formighieri \& Pasian, 2012; Oliveira et al., 2001; Teixeira et al., 2019), retratando marcadores de sua vivência afetiva em diferentes contextos, com indicadores bastante promissores para se compreender características psíquicas e necessidades do envelhecimento. $\mathrm{O}$ estudo mais antigo (Oliveira et al., 2001) comparou achados de entrevista e de escolhas cromáticas e organizações formais do TPC de dois grupos de 25 mulheres com mais de 60 anos, institucionalizadas e não institucionalizadas, no interior do Estado de São Paulo. Identificaram especificidades de produção entre os dois grupos no TPC, mas não diferenças estatisticamente significativas. No entanto, quando compararam cada subgrupo com a expectativa normativa do TPC disponível em adultos na época, identificaram "tendência ao enfraquecimento nos dispositivos de controle da impulsividade (diminuição do azul e preto), acompanhados por sinais sugestivos de ansiedade e de inquietação interna (violeta e cinza aumentados), com possibilidade de descargas diretas no ambiente (amarelo diminuído e branco elevado) e/ou tendências à somatização (violeta elevado) como tentativas de busca de alívio psíquico para as necessidades afetivas insatisfeitas" (p. 79). Essas inferências 
foram possíveis a partir das escolhas e síndromes cromáticas elaboradas pelas idosas desse estudo, o qual evidenciou a relevância dessas variáveis do TPC para a compreensão psicodinâmica, devendo ser objeto de análise em novos trabalhos com esse método projetivo.

Ainda no interior do Estado de São Paulo, BastosFormighieri e Pasian (2012) elaboraram padrões de referência (normas) para o TPC para indivíduos da comunidade (não clínicos) com mais de 60 anos e de ambos os sexos $(n=100)$, procurando particularidades do funcionamento afetivo e cognitivo na velhice. As autoras compararam seus achados com referenciais normativos do TPC para adultos, presentes em Villemor-Amaral (2005). Destacou-se a menor utilização, pelos idosos, de cores de baixa estimulação afetiva, com interpretação de que estes utilizaram menos mecanismos defensivos de repressão, inibição, dissimulação, racionalização, negação e atuação. Os idosos apresentaram diferenças estatisticamente significativas em relação aos adultos normativos nos valores médios das frequências e principais síndromes cromáticas, justificando a elaboração de padrões de referência específicos do TPC para essa faixa etária mais velha. Mais uma vez notou-se a relevância das escolhas e síndromes cromáticas como variáveis centrais para análise e compreensão da psicodinâmica dos participantes, constituindo-se em elementos centrais para novos estudos na área com o TPC.

A partir desse trabalho publicado em 2012, não foi encontrado outro estudo no Brasil voltado ao exame dos padrões de resposta de idosos ao TPC. No entanto, as rápidas e intensas mudanças existentes no contexto sociocultural contemporâneo podem implicar em efeitos nas vivências socioafetivas dos indivíduos. Nesse contexto, torna-se relevante examinar, na atualidade, novos grupos de idosos a partir do TPC, já sabidamente adequado para compreender processos psicodinâmicos dessa faixa etária da população, considerando-se, em especial, as escolhas das cores e a composição das principais síndromes cromáticas (normal, estímulo, fria e incolor) (VillemorAmaral, 2016).

Embora não voltado a caracterização do padrão de respostas de idosos diante do TPC, Teixeira et al. (2019) conduziram estudo com objetivo de investigar o BemEstar Subjetivo (BES) de idosos institucionalizados e não institucionalizados, a partir desse método projetivo de avaliação psicológica. O estudo contou com 70 participantes, com 80 anos ou mais, residentes em cidade do interior do Rio Grande do Sul, divididos em dois grupos em relação à institucionalização (35 institucionalizados e 35 não institucionalizados). Não foram encontradas diferenças estatisticamente significativas entre os dois grupos em relação aos componentes afetivos investigados pelo TPC, mas encontraram pistas de particularidade nas vivências emocionais de idosos, relacionando-as com a condição de moradia (institucionalização).

Apesar da riqueza das informações possíveis com o TPC, além de sua grande aceitação por respondentes idosos, nota-se reduzida produção científica. As evidências empíricas e teóricas anteriormente apontadas sugerem existência de especificidades nas escolhas cromáticas e nas síndromes cromáticas do TPC conforme a etapa do desenvolvimento, mostrando-se necessário realizar investigações adicionais por meio desse método projetivo nessa faixa etária.

Diante do exposto, este estudo objetivou comparar indicadores do funcionamento afetivo derivados do Teste das Pirâmides Coloridas de Pfister (TPC) em idosos institucionalizados e não institucionalizados, tendo em conta que suas condições diferenciadas de moradia poderiam interferir em seu funcionamento psicológico. Considerou-se como hipótese básica a existência de especificidades nas escolhas cromáticas e na composição das síndromes cromáticas do TPC desses dois grupos de idosos, variáveis destacadas nos estudos prévios com esse método projetivo, conforme apontado em BastosFormighieri e Pasian (2012).

\section{Método}

O presente trabalho se constitui enquanto investigação científica com delineamento transversal, caracterizando-se como estudo descritivo-comparativo e interpretativo (Cozby, 2003), realizado com indivíduos idosos. Essa investigação científica foi devidamente examinada e aprovada por um Comitê de Ética em Pesquisa com Seres Humanos (Parecer n. ${ }^{\circ}$ 1.874.029), seguindo-se adequados preceitos éticos em sua realização. Todos os participantes autorizaram livremente sua participação no estudo, incluindo anuência da direção institucional no caso das ILPIs.

\section{Participantes}

Foram considerados como idosos os indivíduos com 60 anos ou mais, segundo critério atual existente no Brasil (Instituto Brasileiro de Geografia e Estatística [IBGE], 2018), sendo inclusos no estudo os voluntários dessa faixa etária, de ambos os sexos, com qualquer nível de escolaridade e estado civil, com capacidade funcional suficiente para responder a questões verbais e para realizar as atividades avaliativas. Foram excluídos idosos com quadros neurológicos degenerativos ou deficiências/perdas sensoriais (Alzheimer, Parkinson, Comprometimento Cognitivo Leve, entre outros).

Foi constituída amostra de conveniência com 50 idosos do interior do Estado de São Paulo, subdivididos em dois grupos a partir de sua condição de moradia: (a) Grupo Clínico (G1, $n=25)$ : residentes em Instituições de Longa Permanência para Idosos (ILPIs), por um período mínimo de seis meses; (b) Grupo de Comparação (G2, $n=25$ ): residentes em seus lares. A idade dos voluntários variou de 61 a 93 anos (média de 73,6 $\pm 8,3$ anos), sendo 24 homens e 26 mulheres, predominando participantes 
com até oito anos de estudo (escolaridade fundamental) e nível econômico médio-inferior (predomínio do nível C no Critério de Classificação Econômica Brasil, CCEB, 2015).

De forma específica, os participantes de G1 tiveram idade entre 62 e 93 anos (média de 77,3 $\pm 8,7$ anos), com tempo médio de institucionalização igual a 3,6 anos $(D P=2,9$ anos). Os idosos de G2 variaram entre $61 \mathrm{e}$ 84 anos (média de 69,9 $\pm 5,9$ anos). A comparação estatística da distribuição etária, de sexo e de escolaridade entre G1 e G2 não apontou diferenças estatisticamente significativas, evidenciando homogeneidade na distribuição dessas características sociodemográficas pelos grupos deste estudo.

\section{Instrumentos}

Os voluntários foram individualmente avaliados por meio do Mini Exame do Estado Mental (MEEM), questionário sociodemográfico (elaborado pelo pesquisador para caracterizar participantes) e Teste das Pirâmides Coloridas de Pfister (TPC). No grupo não institucionalizado (G2), foi ainda utilizado o Critério de Classificação Econômica Brasil (CCEB, 2015).

1. Mini Exame do Estado Mental (MEEM). Proposto por Folstein et al. (1975), foi adaptado ao contexto brasileiro por Chaves e Izquierdo (1992), para população gaúcha, e por Bertolucci et al. (1994), para população paulista. É instrumento de avaliação de funções cognitivas, com ênfase no rastreamento de sintomas de disfunções cognitivas. Neste estudo, foram utilizados os referenciais normativos propostos por Kochhann et al. (2010).

2. Critério de Classificação Econômica Brasil (CCEB, 2015). Sistema de classificação econômico baseado em pontos codificados a partir da quantidade de equipamentos existentes na casa, grau de escolaridade e acesso a serviços públicos. A classificação é feita a partir de níveis (A, B1, B2, C1, C2, D-E), sendo que o nível A corresponde ao mais elevado padrão econômico e o nível $\mathrm{E}$, ao mais baixo padrão econômico, definido a partir da pontuação total no instrumento. O objetivo do uso desse instrumento voltou-se a verificar se haveria similaridade econômica entre G1 (de baixa renda econômica por sua condição de moradia) e os voluntários de G2, possibilitando comparação nas demais variáveis em foco.

3. Questionário sociodemográfico. Questionário aplicado em formato de breve entrevista, para coleta de informações sociodemográficas dos voluntários da pesquisa. Foi elaborado pelo pesquisador, de modo a permitir a identificação de variáveis psicossociais dos participantes, em relação ao sexo, idade e escolarização, variáveis relevantes para o estudo proposto.

4. Teste das Pirâmides Coloridas de Pfister (TPC). Método projetivo de avaliação psicológica, abordando aspectos cognitivos e dinâmica emocional do indivíduo. Os indicadores técnicos do instrumento, no Brasil, apontaram suficientes evidências psicométricas para seu uso em nosso contexto sociocultural (Villemor-Amaral, 2016). Conforme inicialmente proposto, focalizou-se a análise nas variáveis relativas às frequências das cores e das síndromes cromáticas do TPC, dado se mostrarem indicadores relevantes para exame da psicodinâmica socioemocional. Recorreu-se aos referenciais normativos do TPC desenvolvidos por Bastos-Formighieri e Pasian (2012), englobando idosos, para examinar as características específicas dos grupos presentemente compostos.

\section{Procedimentos}

Após a devida autorização institucional das ILPIs, o pesquisador e um profissional de cada instituição realizaram triagem inicial dos casos possíveis para este estudo a partir dos prontuários individuais dos idosos, tendo em vista os critérios de seleção adotados. O primeiro autor fez o convite direto a cada idoso residente na instituição, explicando o estudo, suas atividades e, no caso de aceite, o agendamento para novo contato onde seria realizada a coleta de dados.

Os idosos não institucionalizados foram buscados na comunidade, por indicação de contatos informais dos pesquisadores, após a conclusão da etapa de avaliação de G1, de modo a se buscar o balanceamento dos grupos em termos etários e de sexo. Nesse grupo, houve um primeiro contato telefônico do primeiro pesquisador com os possíveis voluntários, onde explicou a pesquisa em termos gerais e, entre os que concordaram com a proposta, foi agendado dia e horário para visita domiciliar e aplicação dos instrumentos previstos, em ambiente com o devido resguardo ético e técnico.

A coleta de dados foi realizada em sessões individuais, em um único encontro com duração aproximada de 60 minutos. As atividades foram realizadas na seguinte sequência: rapport, autorização do estudo, MEEM, questionário sociodemográfico (com CCEB em G2) e TPC. Após a finalização da coleta dos dados, procedeu-se à codificação deles de acordo com as recomendações técnicas de cada instrumento referidas em seus respectivos manuais ou artigos científicos de referência. Os escores de cada participante, em cada instrumento, foram digitados em planilhas computacionais, diretamente no programa Statistical Package for Social Sciences (SPSS, versão 25.0) possibilitando a elaboração de banco de dados.

A seguir, foram realizadas análises descritivas e inferenciais dos resultados, tendo em vista os objetivos propostos nesta pesquisa. Procurou-se caracterizar os padrões gerais de resposta dos idosos no MEEM e no TPC, bem como a descrição de seu perfil sociodemográfico. A seguir, focalizou-se análise dos achados no TPC, comparando variáveis relativas às escolhas cromáticas (10 cores) e principais síndromes cromáticas (Normal, Estímulo, Fria e Incolor) produzidas por idosos institucionalizados e não institucionalizados entre si e em relação às expectativas normativas disponíveis para essa faixa etária 
(Bastos-Formighieri \& Pasian, 2012). Recorreu-se ao teste $t$ de Student $(p \leq 0,05)$ para essa análise comparativa, acrescida pelo cálculo de tamanho do efeito ( $d$ de Cohen) das diferenças entre os resultados (Cohen, 1988).

\section{Resultados}

A partir da distribuição dos casos de G1 e G2, em relação aos critérios estabelecidos como nota de corte para o MEEM, observou-se que em cada um dos dois grupos, apenas um participante pontuou abaixo dessa nota, estando os demais idosos com desempenho cognitivo compatível ao esperado para sua idade e escolaridade. Apesar das implicações desses achados em relação ao indício de possível declínio cognitivo nesses dois casos, os resultados apontaram homogeneidade na composição de G1 e G2 a partir das informações do MEEM, permitindo análises comparativas entre os grupos nas variáveis selecionadas do TPC.

As análises descritivas e comparativas das médias das frequências cromáticas do TPC dos idosos de G1 e de G2 foram realizadas, não se identificando diferenças estatisticamente significativas entre os grupos. Buscou-se, então, verificar o quanto cada um desses grupos de idosos acompanhava (ou não) o esperado para sua faixa etária em termos de escolhas cromáticas no TPC, comparando os achados aos dados apresentados por Bastos-Formighieri e Pasian (2012), dados que compõem a Tabela 1.

Tabela 1

Análise Descritiva e Comparativa das Frequências Cromáticas do TPC de Idosos Institucionalizados e Não Institucionalizados em Relação a Dados Normativos

\begin{tabular}{|c|c|c|c|c|c|c|c|c|c|c|}
\hline \multirow{2}{*}{$\begin{array}{l}\text { Cores } \\
\text { TPC }\end{array}$} & \multicolumn{2}{|c|}{$\begin{array}{c}\text { Análise descritiva } \\
\text { Média (DP) }\end{array}$} & \multicolumn{3}{|c|}{$\begin{array}{l}\text { Comparação } \\
\text { estatística }\end{array}$} & \multicolumn{2}{|c|}{$\begin{array}{c}\text { Análise descritiva } \\
\text { Média (DP) }\end{array}$} & \multicolumn{3}{|c|}{$\begin{array}{c}\text { Comparação } \\
\text { estatística }\end{array}$} \\
\hline & $\mathrm{G1}^{*}$ & Normas ${ }^{* * *}$ & $t$ & $p$ & $d$ & $\mathrm{G} 2^{* *}$ & Normas ${ }^{* * *}$ & $t$ & $p$ & $d$ \\
\hline Azul & $\begin{array}{l}17,69 \\
(3,98)\end{array}$ & $\begin{array}{l}16,00 \\
(9,00)\end{array}$ & 2,122 & 0,044 & 0,424 & $\begin{array}{l}17,33 \\
(7,69)\end{array}$ & $\begin{array}{l}16,00 \\
(9,00)\end{array}$ & 0,837 & 0,411 & 0,167 \\
\hline Vermelho & $\begin{array}{l}17,78 \\
(6,02)\end{array}$ & $\begin{array}{l}18,50 \\
(7,80)\end{array}$ & $-0,600$ & 0,554 & $-0,120$ & $\begin{array}{c}17,07 \\
(10,20)\end{array}$ & $\begin{array}{l}18,50 \\
(7,80)\end{array}$ & $-0,703$ & 0,489 & $-0,141$ \\
\hline Verde & $\begin{array}{l}18,31 \\
(5,82)\end{array}$ & $\begin{array}{l}19,60 \\
(7,90)\end{array}$ & $-1,107$ & 0,279 & $-0,221$ & $\begin{array}{l}18,49 \\
(8,10)\end{array}$ & $\begin{array}{l}19,60 \\
(7,90)\end{array}$ & $-0,685$ & 0,500 & $-0,137$ \\
\hline Violeta & $\begin{array}{c}9,69 \\
(4,71)\end{array}$ & $\begin{array}{c}7,70 \\
(5,30)\end{array}$ & 2,112 & 0,045 & 0,422 & $\begin{array}{c}8,71 \\
(5,69)\end{array}$ & $\begin{array}{c}7,70 \\
(5,30)\end{array}$ & 0,887 & 0,384 & 0,177 \\
\hline Laranja & $\begin{array}{l}10,22 \\
(5,36)\end{array}$ & $\begin{array}{l}11,90 \\
(7,20)\end{array}$ & $-1,563$ & 0,131 & $-0,313$ & $\begin{array}{c}9,78 \\
(6,22)\end{array}$ & $\begin{array}{l}11,90 \\
(7,20)\end{array}$ & $-1,706$ & 0,101 & $-0,341$ \\
\hline Amarelo & $\begin{array}{c}9,07 \\
(3,62)\end{array}$ & $\begin{array}{l}11,70 \\
(7,00)\end{array}$ & $-3,633$ & 0,001 & $-0,727$ & $\begin{array}{l}11,38 \\
(7,49)\end{array}$ & $\begin{array}{l}11,70 \\
(7,00)\end{array}$ & $-0,215$ & 0,832 & $-0,043$ \\
\hline Marrom & $\begin{array}{c}4,62 \\
(2,39)\end{array}$ & $\begin{array}{c}5,70 \\
(5,20)\end{array}$ & $-2,252$ & 0,034 & $-0,450$ & $\begin{array}{c}6,04 \\
(7,68)\end{array}$ & $\begin{array}{c}5,70 \\
(5,20)\end{array}$ & 0,224 & 0,825 & 0,045 \\
\hline Preto & $\begin{array}{l}2,75 \\
(2,74)\end{array}$ & $\begin{array}{c}2,20 \\
(2,80)\end{array}$ & 1,013 & 0,321 & 0,203 & $\begin{array}{c}3,38 \\
(3,21)\end{array}$ & $\begin{array}{c}2,20 \\
(2,80)\end{array}$ & 1,832 & 0,079 & 0,366 \\
\hline Branco & $\begin{array}{c}7,02 \\
(7,66)\end{array}$ & $\begin{array}{c}4,80 \\
(5,10)\end{array}$ & 1,450 & 0,160 & 0,290 & $\begin{array}{c}5,42 \\
(5,92)\end{array}$ & $\begin{array}{c}4,80 \\
(5,10)\end{array}$ & 0,526 & 0,604 & 0,105 \\
\hline Cinza & $\begin{array}{c}2,84 \\
(2,44)\end{array}$ & $\begin{array}{c}1,50 \\
(2,30)\end{array}$ & 2,750 & 0,011 & 0,550 & $\begin{array}{c}2,40 \\
(3,07) \\
\end{array}$ & $\begin{array}{l}1,50 \\
(2,30)\end{array}$ & 1,465 & 0,156 & 0,293 \\
\hline
\end{tabular}

Nota. ${ }^{*} \mathrm{G} 1=$ grupo institucionalizado $(n=25) ;{ }^{* *} \mathrm{G} 2=$ grupo não institucionalizado $(n=25) ;{ }^{* * *}$ Normas=Bastos-Formighieri e Pasian $(2012$, $n=100)$

A análise comparativa dos resultados médios de G1 com as médias normativas apontou cinco diferenças estatisticamente significativas na frequência cromática das dez cores do TPC. Ocorreram nas seguintes cores: azul, violeta e cinza (média de G1 maior que média normativa, tamanho do efeito pequeno em azul e violeta, moderado em cinza), amarelo e marrom (média de G1 inferior à média normativa, tamanho do efeito moderado em amarelo e pequeno em marrom). Entre G2 e o grupo normativo não foram observadas diferenças estatisticamente significativas. Tem-se, desse modo, escolhas cromáticas similares entre dois grupos de idosos da comunidade, um atualmente avaliado (G2) e outro examinado em 2012, por Bastos-Formighieri e Pasian (2012). 
Na sequência, foi realizada a comparação dos resultados das principais Síndromes Cromáticas (Normal, Estímulo, Fria e Incolor) no TPC, dos dados de G1 e G2, sem identificação de diferenças estatisticamente significativas entre os grupos. Em complemento, examinou-se o quanto cada um desses grupos de idosos seguia o esperado para sua faixa etária na frequência das síndromes cromáticas no TPC, comparando os achados com aqueles apresentados por Bastos-Formighieri e Pasian (2012). Tais resultados estão apresentados na Tabela 2.

Tabela 2

Análise Descritiva e Comparativa das Síndromes Cromáticas do TPC de Idosos Institucionalizados e Não Institucionalizados em Relação a Dados Normativos

\begin{tabular}{|c|c|c|c|c|c|c|c|c|c|c|}
\hline \multirow{2}{*}{$\begin{array}{c}\text { Síndromes } \\
\text { Cromáticas } \\
\text { TPC }\end{array}$} & \multicolumn{2}{|c|}{$\begin{array}{l}\text { Análise descritiva } \\
\text { Média (DP) }\end{array}$} & \multicolumn{3}{|c|}{$\begin{array}{l}\text { Comparação } \\
\text { estatística }\end{array}$} & \multicolumn{2}{|c|}{$\begin{array}{l}\text { Análise descritiva } \\
\text { Média (DP) }\end{array}$} & \multicolumn{3}{|c|}{$\begin{array}{c}\text { Comparação } \\
\text { estatística }\end{array}$} \\
\hline & $\mathrm{G} 1^{*}$ & $\underset{* * *}{\operatorname{Normas}}$ & $t$ & $p$ & $d$ & $\mathrm{G} 2^{* *}$ & $\underset{* * *}{\operatorname{Normas}}$ & $t$ & $p$ & $d$ \\
\hline Normal & $\begin{array}{l}53,78 \\
(7,85)\end{array}$ & $\begin{array}{l}54,10 \\
(9,00)\end{array}$ & $-0,205$ & 0,839 & $-0,041$ & $\begin{array}{l}52,89 \\
(9,12)\end{array}$ & $\begin{array}{l}54,10 \\
(9,00)\end{array}$ & $-0,664$ & 0,513 & $-0,133$ \\
\hline Estímulo & $\begin{array}{l}37,07 \\
(8,28)\end{array}$ & $\begin{array}{c}42,00 \\
(10,70)\end{array}$ & $-2,978$ & 0,007 & $-0,596$ & $\begin{array}{c}38,22 \\
(15,28)\end{array}$ & $\begin{array}{c}42,00 \\
(10,70)\end{array}$ & $-1,237$ & 0,228 & $-0,247$ \\
\hline Fria & $\begin{array}{l}45,69 \\
(7,20)\end{array}$ & $\begin{array}{l}43,30 \\
(9,30)\end{array}$ & 1,658 & 0,110 & 0,332 & $\begin{array}{c}44,53 \\
(12,43)\end{array}$ & $\begin{array}{l}43,30 \\
(9,30)\end{array}$ & 0,496 & 0,624 & 0,099 \\
\hline Incolor & $\begin{array}{l}12,62 \\
(7,53)\end{array}$ & $\begin{array}{c}8,50 \\
(7,00)\end{array}$ & 2,738 & 0,011 & 0,548 & $\begin{array}{l}11,20 \\
(7,60)\end{array}$ & $\begin{array}{c}8,50 \\
(7,00)\end{array}$ & 1,776 & 0,088 & 0,355 \\
\hline
\end{tabular}

Nota. ${ }^{*} \mathrm{G} 1=$ grupo institucionalizado $(n=25) ;{ }^{* *} \mathrm{G} 2=$ grupo não institucionalizado $(n=25) ;{ }^{* * *}$ Normas=Bastos-Formighieri e Pasian $(2012$, $n=100$ )

Em relação a G1, a análise apontou diferenças estatisticamente significativas em relação à Síndrome Estímulo (SE) e à Síndrome Incolor (SI). A SE, composta pela soma da frequência das cores vermelha, laranja e amarela, apresentou média mais elevada no grupo normativo do que em G1. Em relação à Síndrome Incolor, observou-se o contrário, sendo que G1 apresentou média superior ao grupo de referência. A SI é composta pela soma da frequência das cores: preto, branco e cinza. O tamanho do efeito, para ambas as diferenças estatisticamente significativas encontradas, foi moderado.

No tocante a $G 2$, os dados não apontaram diferenças estatisticamente significativas nas principais Síndromes Cromáticas do TPC em relação ao esperado para essa faixa etária. Esses dois grupos de idosos da comunidade (G2 e grupo normativo de 2012) apresentaram frequências similares nas principais síndromes cromáticas do TPC, mesmo sendo examinados em épocas distintas.

\section{Discussão}

Para iniciar o processo de reflexão sobre os dados, faz-se relevante retomar o objetivo delineado para esse trabalho. Almejou-se comparar indicadores de vivências afetivas de idosos institucionalizados e de idosos não institucionalizados, a partir das escolhas cromáticas e síndromes cromáticas do TPC, variáveis classicamente reconhecidas como adequados representantes da psicodinâmica socioemocional (Villemor-Amaral, 2016).
Os resultados, no entanto, não confirmaram essa hipótese inicial de especificidades nessas escolhas das cores e síndromes cromáticas por parte desses dois grupos de idosos, relativizando o efeito da condição de moradia sobre suas vivências afetivas, segundo as variáveis examinadas no TPC.

No entanto, a análise comparativa da frequência de uso das cores e da composição das síndromes cromáticas de G1 e G2 em relação aos dados normativos do TPC para idosos, elaborados por Bastos-Formighieri e Pasian (2012), evidenciou diferenças estatisticamente significativas. Ou seja, emergiram especificidades nos indicadores relativos a vivências afetivas de idosos institucionalizados e não institucionalizados, sendo necessário buscar compreendê-las do ponto de vista técnico e em seu significado clínico, a partir da literatura científica disponível.

É importante destacar que dessa comparação dos atuais resultados nas escolhas e síndromes cromáticas do TPC em relação às normas disponíveis para idosos (Bastos-Formighieri \& Pasian, 2012) resultaram diferenças estatisticamente significativas apenas em G1. Desse modo, têm-se pistas de marcadores de particularidades nas vivências afetivas de idosos institucionalizados em relação aqueles que residem em seus lares (G2 e grupo normativo).

Em relação ao uso das cores (frequência cromática), retoma-se que as diferenças estatisticamente significativas encontradas deram-se apenas em relação ao azul, ao violeta e ao cinza, tendo G1 média superior ao grupo 
normativo. Segundo Villemor-Amaral (2016), o azul seria a cor relacionada ao controle dos afetos e, quando aumentado, traria um sentido interpretativo de sentimento de inferioridade, de incapacidade, insatisfações e ambivalência, sendo que o indivíduo que apresenta aumento nessa cor seria menos espontâneo no contato com o ambiente, apresentando rigidez e comportamentos mais estereotipados. Por sua vez, a cor violeta é tradicionalmente associada à ansiedade, enquanto o cinza carrega significado de carência afetiva e sentimento de vazio, assim como ansiedade, insegurança e repressão dos afetos. Seu uso aumentado, como evidenciado nos dados de idosos institucionalizados deste trabalho, indicaria retraimento defensivo, no sentido de restrição nos contatos emocionais (Villemor-Amaral, 2016).

As outras cores que apresentaram diferenças estatisticamente significativas, na comparação de G1 para com os dados normativos, foram o amarelo e o marrom (G1 inferior à média normativa). $\mathrm{O}$ amarelo estaria associado, segundo Villemor-Amaral (2016), a uma extroversão mais adaptada, sendo que, quando diminuído, indicaria dificuldade em expressar emoções de forma ajustada ao ambiente. O marrom, quando diminuído, sinalizaria falta de energia (ou menor resistência) e baixa produtividade.

Em relação às Síndromes Cromáticas, foi possível observar em G1 diminuição da Síndrome Estímulo e aumento significativo da Síndrome Incolor. A Síndrome Estímulo é constituída pelas cores mais estimulantes (vermelho, amarelo, laranja), enquanto a Síndrome Incolor estaria associada aos tons de neutralidade ou acromáticos (preto, branco e cinza). A partir de seus significados simbólicos (Villemor-Amaral, 2016), reforça-se o sentido de que os idosos institucionalizados (G1) avaliados neste trabalho sinalizaram indícios de maior continência afetiva e mais sentimentos ansiogênicos quando comparados com seu grupo normativo, com menor disponibilidade para vivenciar seus conteúdos afetivos de forma adaptada ao ambiente no qual se inserem.

Diante dessas possibilidades interpretativas dos atuais resultados do TPC em idosos, evidenciaram-se especificidades nas vivências emocionais de G1, imprimindo um caráter mais negativo nessas experiências. Seria precoce apontar qualquer variável como definidora dessa diretriz emocional nos idosos, sobretudo porque ainda os estudos com essa faixa etária, tanto no Brasil quanto no mundo, pouco exploraram eventuais marcadores em termos afetivos (Lamas et al., 2018; Martins et al., 2019).

Além disso, cabe destacar que os idosos em condições semelhantes de moradia (G2 e referenciais normativos) apresentaram forma semelhante de responder às variáveis de escolhas e síndromes cromáticas do TPC. Há evidências empíricas, portanto, da estabilidade temporal dos achados do TPC com idosos da comunidade, comparando-se amostras avaliadas em duas épocas diferentes. Desse modo, poder-se-á refletir sobre as variáveis envolvidas na institucionalização e possíveis intervenções ou planejamento futuro de novas abordagens nas próprias atividades cotidianas das ILPIs de modo a favorecer a preservação e o aproveitamento dos recursos internos na velhice.

Ao examinar o bem-estar subjetivo de longevos institucionalizados e não institucionalizados por meio do TPC na região Sul do Brasil, Teixeira et al. (2019) não identificaram especificidades nas escolhas e nas síndromes cromáticas dos idosos. Seus indicadores no TPC mostraram similaridade, independentemente de sua condição de moradia. Nesse estudo, foram investigadas outras variáveis, incluindo depressão, afetos positivos e negativos e autoestima, por meio de outros recursos técnicos de avaliação psicológica. A única diferença estatisticamente significativa identificada entre esses grupos ocorreu no tocante aos afetos positivos, conforme avaliação do instrumento PANAS, sendo que os idosos institucionalizados evidenciaram menor intensidade dessas vivências quando comparados aos não institucionalizados. Ainda que seus achados não tenham ocorrido no TPC, como neste atual trabalho, encontraram pistas de particularidade nas vivências emocionais de idosos, relacionando-as com a condição de moradia (institucionalização). No caso, sinalizaram menos afetos positivos entre institucionalizados.

O bem-estar subjetivo de idosos institucionalizados e não institucionalizados também foi objeto de investigação no interior do Estado de São Paulo, com uso do TPC (Oliveira et al., 2001). Nessa época, foram examinadas apenas mulheres $(n=50)$, metade em cada grupo (asiladas e não asiladas), destacando achados de entrevista e de suas escolhas cromáticas e organizações formais dos estímulos do TPC. Houve indícios sugestivos de diminuição no uso de mecanismos racionais de controle e de introversão nessa fase do desenvolvimento, associados a preservação da sensibilidade ao meio e sinais de ansiedade. Observaram, também, tendência ao enfraquecimento no controle da impulsividade, com possibilidade de descargas diretas no ambiente e/ou tendências à somatização, como tentativas de busca de alívio psíquico para as necessidades afetivas. De forma similar, essas características foram identificadas em G1 do atual estudo, com destaque para aumento de violeta e cinza, sugerindo indicadores de vivências de inquietação psíquica entre idosos moradores em ILPIs. Apesar desses pontos de proximidade, há que se comentar que as amostras são reduzidas em ambos os estudos, exigindo novas investigações, incluindo também outras regiões do Brasil.

A partir da articulação dos achados atuais com a literatura científica voltada ao exame da afetividade a partir do TPC, reforça-se a necessidade do avanço contínuo dos estudos em Psicologia relacionados ao processo de envelhecimento (Cachioni et al., 2017), por sua premência na realidade contemporânea. Destaca-se o uso de instrumentos de avaliação psicológica nessas 
investigações científicas (Fonseca et al., 2014; Fontes et al., 2015; Gonçalves, 2014) e, de forma mais específica, a possibilidade de aplicação de métodos projetivos (Moura \& Lopes, 2017; Reichert \& Scortegagna, 2016; Teixeira et al., 2019), nos quais se insere o TPC, visando a compreensão da dinâmica afetiva do indivíduo (Silva \& Cardoso, 2012).

\section{Considerações Finais}

Dentre as possibilidades deste trabalho, foi possível apontar particularidades do processo de envelhecimento aliado a uma condição de moradia específica que é a institucionalização. Mais do que isso, o trabalho apontou que, em condições semelhantes de moradia, os idosos de G2 (atual trabalho) e os idosos que compuseram a amostra normativa (Bastos-Formighieri \& Pasian, 2012) demonstraram formas semelhantes de vivenciar sua afetividade, a partir das evidências das escolhas cromáticas e síndromes cromáticas do TPC. Desse modo, pode-se contribuir para o avanço e para reflexões no campo da avaliação dos afetos em indivíduos idosos com métodos projetivos. Destacam-se as qualidades práticas e lúdicas do TPC (Villemor-Amaral, 2016) como recurso técnico promissor, sobretudo diante do crescente envelhecimento populacional na contemporaneidade, além das formas para cuidar desses indivíduos em termos de saúde, ressaltando a relevância do avanço em investigações relativas aos possíveis efeitos da institucionalização, nessa etapa do desenvolvimento.

\section{Agradecimentos}

Agradecemos aos idosos participantes deste estudo que conosco compartilharam suas vivências e histórias de vida e às Instituições de Longa Permanência de Idosos (ILPI) que gentilmente abriram suas portas para esta pesquisa.

\section{Financiamento}

A presente pesquisa não recebeu nenhuma fonte de financiamento sendo custeada com recursos dos próprios autores.

\section{Contribuições dos autores}

Declaramos que todos os autores participaram da elaboração do manuscrito. Especificamente, os autores Rafael Lima Dalle Mulle e Sonia Regina Pasian participram da redação inicial do estudo - conceitualização, investigação, visualização, da análise dos dados e da redação final do trabalho - revisão e edição.

\section{Disponibilidade dos dados e materiais}

Todos os dados e sintaxes gerados e analisados durante esta pesquisa serão tratados com total sigilo devido às exigências do Comitê de Ética em Pesquisa em Seres Humanos. Porém, o conjunto de dados e sintaxes que apoiam as conclusões deste artigo estão disponíveis mediante razoável solicitação ao autor principal do estudo.

\section{Conflito de interesses}

Os autores declaram que não há conflitos de interesses.

\section{Referências}

Agência Nacional de Vigilância Sanitária (ANVISA) (2017). Recuperado de http://portal.anvisa.gov.br/

Alves-Silva, J. D., Scorsolini-Comin, F., \& Santos, M. A. D. (2013). Idosos em instituições de longa permanência: Desenvolvimento, condições de vida e saúde. Psicologia: Reflexão e Crítica, 26(4), 820-830. http://dx.doi.org/10.1590/S0102-79722013000400023

Bastos-Formighieri, M. S., \& Pasian, S. R. (2012). O Teste Pfister em Idosos. Avaliação Psicológica, 11(3), 435-448. Recuperado de http:// www.redalyc.org/html/3350/335027503010/

Bertolucci, P. H. F., Brucki, S. M. D., Campacci S., \& Juliano, Y. (1994). O Mini-Exame do Estado Mental em uma população geral: Impacto da escolaridade. Arquivos de Neuropsiquiatria, 52(1), 1-7. http://dx.doi.org/10.1590/S0004-282X1994000100001

Birren, J. E., \& Schaie, K. W. (2006). Handbook of the psychology of aging (6th ed.). San Diego, CA: Elsevier Academic Press.

Cachioni, M., Delfino, L. L., Yassuda, M. S., Batistoni, S. S. T., Melo, R. C., \& Domingues, M. A. R. C. (2017). Bem-estar subjetivo e psicológico de idosos participantes de uma Universidade Aberta à Terceira Idade. Revista Brasileira de Geriatria e Gerontologia, 20(3), 340352. http://dx.doi.org/10.1590/1981-22562017020.160179

Chaves, M. L. F., \& Izquierdo, I. (1992). Differential diagnosis between dementia and depression: a study of efficiency increment. Acta Neurologica Scandinavica, 85(6), 378-382. http://dx.doi.org/10.1111/j.1600-0404.1992.tb06032.x

Cohen, J. (1988). Statistical Power Analysis for the Behavioral Sciences (2nd ed.). New York: Lawrence Erlbaum Associates, Publishers.

Costa, M. C. N. S., \& Mercadante, E. F. (2013). O Idoso residente em ILPI (Instituição de Longa Permanência do Idoso) e o que isso representa para o sujeito idoso. Revista Kairós: Gerontologia, 16(1), 209-222. Recuperado de https://revistas.pucsp.br/kairos/article/ view/17641/13138

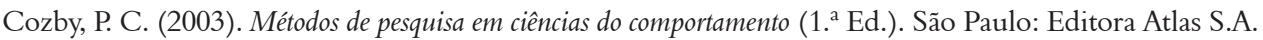

Critério de Classificação Econômica Brasil (2015). Alterações na aplicação do Critério Brasil, válidos a partir de 01/01/2015. Recuperado de http:// www.abep.org/

Folstein, M. F., Folstein, S. E., \& McHugh, P. R. (1975). Mini-Mental State. A practical method for grading the cognitive state of patients for the clinician. Journal of Psychiatry Research, 12(3), 189-198. http://dx.doi.org/10.1016/0022-3956(75)90026-6

Fonseca, C. C., Chaves, É. D. C. L., Pereira, S. S., Barp, M., Moreira, A. M., \& Nogueira, D. A. (2014). Autoestima e satisfação corporal em idosas praticantes e não praticantes de atividades corporais. Journal of Physical Education, 25(3), 429-439. http://dx.doi.org/10.4025/reveducfis.v25i3.22050 
Fontes, A. P., Fattori, A., D’Elboux, M. J., \& Guariento, M. E. (2015). Resiliência psicológica: Fator de proteção para idosos no contexto ambulatorial. Revista Brasileira de Geriatria e Gerontologia, 18(1), 7-17. http://dx.doi.org/10.1590/1809-9823.2015.13201

Gonçalves, I. F. (2014). Instrumentos de Avaliação Psicológica em Idosos. [Dissertação de mestrado, Instituto Superior Miguel Torga - Coimbra]. Recuperado de http://repositorio.ismt.pt/handle/123456789/626

Guimarães, L. D. A., Brito, T. A., Pithon, K. R., Jesus, C. S. D., Souto, C. S., Souza, S. J. N., \& Santos, T. S. D. (2019). Sintomas depressivos e fatores associados em idosos residentes em instituição de longa permanência. Ciência E Saúde Coletiva, 24(9), 3275-3282. http:// dx.doi.org/10.1590/1413-81232018249.30942017

Instituto Brasileiro de Geografia e Estatística (IBGE, 2018). Recuperado de www.ibge.gov.br/home/

Kochhann, R., Varela, J. S., Lisboa, C. S. M., \& Chaves, M. L. F. (2010). The Mini Mental State Examination: Review of cutoff points adjusted for schooling in a large Southern Brazilian sample. Dementia e Neuropsychologia, 4(1), 35-41. http://dx.doi.org/10.1590/S198057642010DN40100006

Lamas, M. F. M., Schettino, N. M. A., Barroso, A. P. N. B., Santos, J. A., \& Banhato, E. F. C. (2018). Perfil cognitivo, funcional e de humor de uma amostra comunitária de idosos. ANALECTA-Centro de Ensino Superior de Juiz de Fora, 4(4), 549-567. Recuperado de https://seer. cesjf.br/index.php/ANL/article/viewFile/1794/1139

Lima, A. A. C., Camargo, A., Raulik, C. G., Campos, D. B., \& Pereira, W. M (2017). Formas metodológicas de avaliação em idosos institucionalizados: Uma revisão sistemática. Cinergis, 18(3), 240-247. http://dx.doi.org/10.17058/cinergis.v18i3.8328

Martins, N. I. M., Caldas, P. R., Cabral, E. D., Lins, C. C. D. S. A., \& Coriolano, M. D. G. W. D. (2019). Instrumentos de avaliação cognitiva utilizados nos últimos cinco anos em idosos brasileiros. Ciência \& Saúde Coletiva, 24(7), 2513-2530. http://dx.doi.org/10.1590/141381232018247.20862017

Menezes, K. M. G., \& Frota, M. H. P. (2012). O lazer enquanto expressão de vitalidade na velhice: a experiência de um centro de convivência de idosos em Fortaleza - CE. Revista Brasileira de Sociologia da Emoção, 11(32), 486-502.

Miguel, F. K., Zuanazzi, A. C., \& Villemor-Amaral, A. E. (2017). Avaliação de aspectos da inteligência emocional nas técnicas de Pfister e Zulliger. Temas em Psicologia, 25(4), 1853-1862. http://dx.doi.org/10.9788/TP2017.4-17Pt

Moura, G. C., \& Lopes, A. A. (2017). O uso de instrumentos projetivos no processo de avaliação psicológica. Caderno de Graduação-Ciências Humanas e Sociais-UNIT-ALAGOAS, 4(2), 53-62. Recuperado de https://periodicos.set.edu.br/index.php/fitshumanas/article/ view/4175

Neri, A. L., Yassuda, M. S., Araújo, L. F., Eulálio, M. C., Cabral, B. E., Siqueira, M. E. C., Santos, G. A., \& Moura, J. G. A. (2013). Metodologia e perfil sociodemográfico, cognitivo e de fragilidade de idosos comunitários de sete cidades brasileiras: Estudo FIBRA. Caderno de Saúde Pública, 29(4), 778-792. http://dx.doi.org/10.1590/S0102-311X2013000400015

Nicolodi, F., \& Scortegagna, S. A. (2012). As Pirâmides Coloridas de Pfister e a afetividade em idosos institucionalizados. Em Scortegagna, S.A. (Ed.). Avaliação Psicológica: expressão singular em diversos contextos (pp. 120-146). 1ed. Passo Fundo (RS): UPF Editora.

Oliveira, E. A., Pasian, S. R., \& Jacquemin, A. (2001). A vivência afetiva em idosos. Psicologia: Ciência e Profissão, 21(1), 68-83. http://dx.doi. org $/ 10.1590 /$ S1414-98932001000100008

Reichert, C., \& Scortegagna, S. A. (2016). Técnicas projetivas com idosos: Revisão de literatura de artigos publicados no Brasil. Métodos projetivos e suas demandas na psicologia contemporânea - Associação Brasileira de Rorschach e Métodos Projetivos (ASBRo), pp. 632-642. Recuperado de https://www.asbro.org.br/arquivos/Metodos_projetivos_e_suas_demandas_na_Psicologia_Contemporanea_Livro_CD_VIII_ Cong_ASBRo_2016.pdf

Silva, L. M., \& Cardoso, L. C. (2012). Revisão de pesquisas brasileiras sobre o teste de Pfister. Avaliação Psicológica, 11(3), 449-460. Recuperado de http://www.redalyc.org/html/3350/335027503011/

Souza, R. C. F., \& Inácio, A. N. (2017). Entre os muros do abrigo: Compreensões do processo de institucionalização em idosos abrigados. Revista Pesquisas e Práticas Psicossociais, 12(1), 209-223. Recuperado de http://seer.ufsj.edu.br/index.php/revista_ppp/article/view/1915

Teixeira, C. R., Scortegagna, S. A., Pasian, S. R., \& Portella, M. R. (2019). Bem-estar subjetivo de longevos institucionalizados e não institucionalizados por meio do Pfister. Avaliação Psicológica, 18(1), 86-95. http://dx.doi.org/10.15689/ap.2019.1801.13512.10

Villemor-Amaral, A. E. (2005). As Pirâmides Coloridas de Pfister. CETEPP.

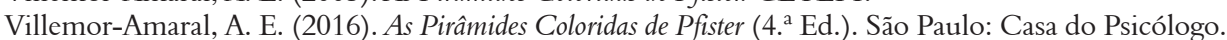

World Health Organization (WHO, 2015). Relatórios Mundiais de Saúde. Recuperado de www.who.int/eportuguese/publications/pt/

\section{Sobre os autores}

Rafael Lima Dalle Mulle é psicólogo e mestre em Psicologia pela Faculdade de Filosofia, Ciências e Letras da Universidade de São Paulo.

Sonia Regina Pasian é psicóloga e professora titular do Departamento de Psicologia da Faculdade de Filosofia, Ciências e Letras de Ribeirão Preto, Universidade de São Paulo.

\section{Como citar este artigo}

Mulle, R. L. D., \& Pasian, S. R. (2021). Envelhecimento e Afetividade a partir do Teste de Pfister. Avaliação Psicológica, 20(1), 80-88. http:// dx.doi.org/10.15689/ap.2021.2001.19555.09 